\title{
On the use of a loudspeaker for measuring the viscoelastic properties of sound absorbing materials
}

\author{
Olivier Doutres, Nicolas Dauchez, Jean-Michel Génevaux, and Guy Lemarquand \\ LAUM, CNRS, Université du Maine, Av. O. Messiaen, 72095 Le Mans, France \\ olivier.doutres@univ-lemans.fr.nicolas.dauchez@univ-lemans.fr. \\ jean-michel.genevaux@univ-lemans.fr,guy.lemarquand@univ-lemans.fr
}

\begin{abstract}
This paper investigates the feasibility to use an electrodynamic loudspeaker to determine viscoelastic properties of sound-absorbing materials in the audible frequency range. The loudspeaker compresses the porous sample in a cavity, and a measurement of its electrical impedance allows one to determine the mechanical impedance of the sample: no additional sensors are required. Viscoelastic properties of the material are then estimated by inverting a 1D Biot model. The method is applied to two sound-absorbing materials (glass wool and polymer foam). Results are in good agreement with the classical compression quasistatic method.
\end{abstract}

(C) 2008 Acoustical Society of America

PACS numbers: 43.20.Ye, 43.20.Jr, 43.38.Dv [MS]

Date Received: August 1,2008 Date Accepted: September 20, 2008

\section{Introduction}

Characterization of sound-absorbing materials, like mineral wool or polymer foam, in the context of building or transport applications, requires the determination of viscoelastic properties of the skeleton. ${ }^{1}$ Classical methods to measure viscoelastic properties of porous materials can be sorted in two groups: the quasistatic methods neglect the inertial effects of the frame and give relevant information in the low-frequency range before the first resonance of the system ${ }^{2,3}$ (usually for $f<100 \mathrm{~Hz}$ ); the dynamic methods are based on the vibration of a porous sample, ${ }^{4}$ or of a structure which includes a porous layer, ${ }^{5}$ and give information at the resonance frequencies of the structure. More recently, methods based on the propagation on surface waves at the free surface of a porous layer have been investigated. ${ }^{6,7}$ These methods require the use of an actuator, usually a shaker, and specific sensors: accelerometer, force sensor, or laser vibrometer [Fig. $1(\mathrm{a})]$.

This paper investigates an alternative method of the classical compression quasistatic setup to measure the viscoelastic properties of porous material. The method is based on the use of an electrodynamic loudspeaker as actuator and sensor. ${ }^{8}$ Since no specific sensors are required, the experimental setup is simplified. Moreover, designed to radiate in the audible frequency range, a loudspeaker has the ability to excite the porous sample in the relevant frequency range for noise control applications. The proposed method is described in Fig. 1(b): the porous sample is set in a cavity and compressed by an electrodynamic loudspeaker. Measurement of the electrical impedance of the loudspeaker allows one to determine the mechanical impedance of the sample by inverting an electroacoustic model. The viscoelastic properties of the porous frame are then estimated using the classical Biot model. ${ }^{9,10}$.

The first part of the paper presents the experimental setup. The principle of measurement and the poroelastic model used for the determination of the viscoelastic properties are then described. Results for two porous materials are finally compared with the classical compression quasistatic method of Fig. 1(a).

\section{Measurement setup}

The experimental setup to validate the proposed electrodynamic technique is presented in Fig. 1(b). A loudspeaker of $71 \mathrm{~mm}$ diameter is mounted between two cavities and applies static and 


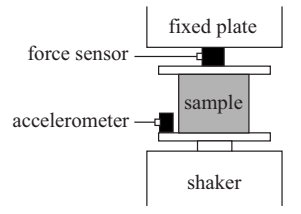

(a)

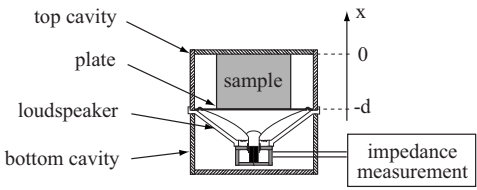

(b)

Fig. 1. Measurement setup: (a) classical compression quasistatic method; (b) new method using an electrodynamic loudspeaker.

dynamic strains to a porous sample set in the top cavity. The sample diameter is smaller than the one of the cavity to avoid any lateral strain. The depth of the cavity can be adjusted to the size of the porous sample to impose a static compression (see Table 2 ). The cavity is used to simplify the inversion procedure and to limit the effect of air pumping. ${ }^{3}$

A circular aluminum plate of $1 \mathrm{~mm}$ thickness is bonded on the loudspeaker cone to ensure a planar and unidirectional compression of the porous sample. The association of the plate and the cone is called the diaphragm. It has its first resonance frequency around $2 \mathrm{kHz}$ and is considered to behave as a rigid body far below this frequency.

Measurement of the electrical impedance is performed with a precision magnetic analyzer (Wayne Kerr PMA 3260A) using a sine step signal from 25 to $200 \mathrm{~Hz}$.

\section{Principal of measurement}

\subsection{Determination of the sample mechanical impedance}

The equivalent electroacoustic circuit given by Thiele and Small ${ }^{11}$ is used to model the loudspeaker. This low-frequency model is valid below the first structure resonance frequency of the loudspeaker diaphragm. Figure 2 is the analogous circuit for the setup of Fig. 1(b), with $U$ the output voltage of the source, $r_{e}$ the dc resistance of the voice coil, $L_{e}$ the inductance of the voice coil, $r_{f}$ a shunting parallel resistance which accounts for eddy current loss in the pole piece, $B$ the magnetic flux density in the air gap, $l$ the length of the voice coil in the magnetic field, $R_{m s}$ the mechanical resistance of the driver suspension losses, $M_{m s}$ the mass of the diaphragm including voice coil, $C_{m s}$ the compliance of the loudspeaker suspensions, $Z_{2 a}$ the mechanical impedance of the bottom air cavity, $Z_{1 a}$ the mechanical impedance of the top air cavity surrounding the porous sample, and $Z_{1 p}$ the mechanical impedance of the porous sample.

Mechanical impedance is defined by the ratio of the force $F$ applied to the diaphragm on its velocity $v$. In the considered frequency range, the two air cavities can be considered as simple compliances and the corresponding mechanical impedances are given by

$$
Z_{1 a}=\frac{S^{\prime 2}}{j \omega\left(V_{1 a} / \rho_{0} c^{2}\right)}, \quad Z_{2 a}=\frac{S^{2}}{j \omega\left(V_{2 a} / \rho_{0} c^{2}\right)},
$$

where $j$ is the square root of $-1, \omega$ the circular frequency of the excitation, $\rho_{0}$ the air density and $c$ the velocity of sound in air, $V_{1 a}$ and $V_{2 a}$ the volume of air in the top and bottom cavity respec-

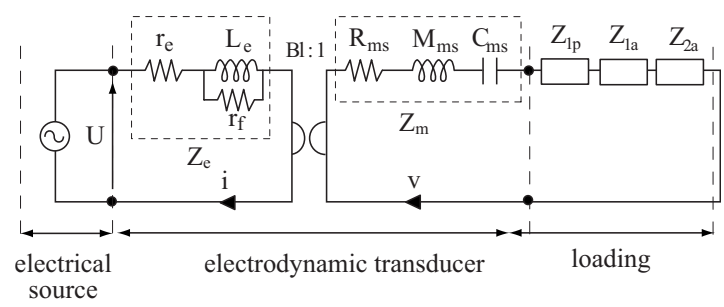

Fig. 2. Electrical equivalent circuit of the measurement setup. 
tively, $S$ the equivalent surface area of the diaphragm in contact with the bottom cavity, and $S^{\prime}$ the equivalent surface area of the upper face of the diaphragm in contact with the air layer in the top cavity.

The electrical impedance of the circuit (Fig. 2) is written

$$
Z_{v c}=\frac{U}{i}=Z_{e}+\frac{(B l)^{2}}{Z_{m}+\left[Z_{1 p}+Z_{1 a}+Z_{2 a}\right]}
$$

with

$$
Z_{e}=r_{e}+\frac{j \omega L_{e} r_{f}}{j \omega L_{e}+r_{f}}
$$

and

$$
Z_{m}=R_{m s}+j \omega M_{m s}+\frac{1}{j \omega C_{m s}} .
$$

Hence, the mechanical impedance of the sample can be derived from the measurement of $Z_{v c}$, the properties of the loudspeaker and of the two air cavities, as

$$
Z_{1 p}=\frac{(B l)^{2}}{Z_{v c}-Z_{e}}-\left(Z_{m}+Z_{1 a}+Z_{2 a}\right) .
$$

\subsection{Determination of the loudspeaker properties}

The properties of the loudspeaker are determined from the measurement of the electrical impedance without porous sample in the top cavity. In that case, the equivalent electrical circuit model gives

$$
Z_{v c}=Z_{e}+\frac{(B l)^{2}}{Z_{m}+\left[Z_{1 a}+Z_{2 a}\right]},
$$

with $Z_{1 a}=S^{2} \rho_{0} c^{2} / j \omega V_{1 a}$ and $V_{1 a}$ the volume of the top cavity. The model is fitted on the measurement using a nonlinear least-squares method to get $r e, L e, r_{f}, B l, R_{m s}, C_{m s}$, and $M_{m s}$.

\subsection{Calculation of the material viscoelastic properties}

The viscoelastic properties of the porous frame are estimated from the impedance $Z_{1 p}$ by the inverse method using the Biot model. In the considered model, the material is isotropic and the displacements of the frame and air are one-dimensional along the sample thickness $[x$ direction in Fig. 1(b)]. The porous material is considered as infinite in the lateral directions and the effects of the boundary conditions are neglected. This assumption is valid for porous materials such as glass wool with a Poisson's ratio equal to 0 , but it can induce an evaluation discrepancy for foam materials which can bulge sideways when compressed between two rigid plates. ${ }^{12}$

The mechanical impedance can be derived analytically from the calculation of the total stress $\left(\sigma_{x x}^{t}\right)$ applied by the porous sample to the vibrating diaphragm,

$$
Z_{1 p}^{\text {th }}(\omega)=\frac{F}{v}=\frac{S_{p}^{\prime \prime} \sigma_{x x}^{t}}{j \omega u_{w}},
$$

with $S_{p}$ the surface area of the porous sample in contact with the vibrating diaphragm $\left(S_{p}=S\right.$ $\left.-S^{\prime}\right)$ and $u_{w}$ the amplitude of the displacement imposed by the diaphragm. According to Biot theory, ${ }^{9,10}$ two compressional waves propagate in a porous media having a one-dimensional behavior. These waves are characterized by a complex wave number $\delta_{i}(i=1,2)$ and a displacement ratio $\mu_{i}$. The total stress exerted by the sample to the diaphragm is thus the sum of the stress exerted by the fluid and solid phases characterized by these two waves as 
Table 1. Properties of the loudspeaker.

\begin{tabular}{ccccccc}
\hline \hline $\begin{array}{c}M_{m s} \\
(\mathrm{~g})\end{array}$ & $\begin{array}{c}C_{m s} \\
\left(\mu \mathrm{m} \mathrm{N}^{-1}\right)\end{array}$ & $\begin{array}{c}R_{m s} \\
\left(\mathrm{~N} \mathrm{~s} \mathrm{~m}^{-1}\right)\end{array}$ & $\begin{array}{c}B l \\
\left(\mathrm{~N} \mathrm{~A}^{-1}\right)\end{array}$ & $\begin{array}{c}r_{e} \\
(\Omega)\end{array}$ & $\begin{array}{c}L_{e} \text { at } 100 \mathrm{~Hz} \\
(\mu \mathrm{H})\end{array}$ & $\begin{array}{c}r_{f} \text { at } 100 \mathrm{~Hz} \\
(\Omega)\end{array}$ \\
\hline \hline 9.37 & 484 & 1.05 & 4.93 & 12.03 & 878 & 2.93 \\
\hline & & & & & \\
$\sigma_{x x}^{t}$ & $=$ & $\sigma_{x x}^{s}+\sigma_{x x}^{f}$ & \\
& $=\left[(\tilde{P}+\tilde{Q})+\mu_{1}(\tilde{R}+\tilde{Q})\right] \delta_{1} \cos \left(\delta_{1} d\right) D_{1}+\left[(\tilde{P}+\tilde{Q})+\mu_{2}(\tilde{R}+\tilde{Q})\right] \delta_{2} \cos \left(\delta_{2} d\right) D_{2}$.
\end{tabular}

In these equations, $d$ is the sample thickness, $\tilde{P}$ and $\tilde{R}$ are the bulk modulus of the solid and fluid phases, respectively, and $\tilde{Q}$ quantifies the potential coupling between the two phases. The expression for these two last coefficients can be found in Ref. 10. Note that viscoelastic properties of the frame, Young's modulus $E$, and loss factor $\eta$, are present in the expression of $\tilde{P}$,

$$
\tilde{P}=\frac{E(1+j \eta)(1-\nu)}{(1+\nu)(1-2 \nu)}+\frac{\tilde{Q}^{2}}{\tilde{R}} .
$$

$D_{1}$ and $D_{2}$ are the amplitude coefficients of the two compressional waves and can be determined from the boundary conditions applied to the sample. Here, the displacement is zero at $x=0$ and is equal to the one of the diaphragm at $x=-d$, which gives

$$
D_{1}=\frac{u_{w}\left(\mu_{2}-1\right)}{\sin \left(\delta_{1} d\right)\left(\mu_{1}-\mu_{2}\right)}, \quad D_{2}=\frac{u_{w}\left(1-\mu_{1}\right)}{\sin \left(\delta_{2} d\right)\left(\mu_{1}-\mu_{2}\right)} .
$$

\section{Results}

The properties of the loudspeaker determined from the measurement of the electrical impedance when the top cavity is empty [see Eq. (6)] are given in Table 1 . Note that the inductance $L_{e}$ decreases and the shunt resistance $r_{f}$ increases with frequency because of the eddy currents flowing in the iron pole structure. ${ }^{13}$ These properties have thus been determined according to the frequency and only the values at $100 \mathrm{~Hz}$ are given in Table 1 .

Measurements of the electrical impedance $Z_{v c}$ are then carried out with a light glass wool and a stiff polymer foam placed in the top cavity. The properties of the materials given in Table 2 have been measured in our laboratory.

The mechanical impedance determined from Eq. (5) and the simulations derived from Eq. (7) are shown in Fig. 3. The Young's moduli used in the model have been measured at $5 \mathrm{~Hz}$ using the compression quasistatic method $^{2}$ (see the measurements Fig. 4). Note that the Young's

Table 2. Properties of materials A and B.

\begin{tabular}{lcc}
\hline \hline Porous & Glass wool A & Foam B \\
\hline Nominal thickness: $d(\mathrm{~mm})$ & 63 & 40.7 \\
Static compression rate $(\%)$ & 37 & 0.95 \\
Sample diameter $(\mathrm{mm})$ & 57 & 44.5 \\
Airflow resistivity: $\sigma\left(\mathrm{kN} \mathrm{s} / \mathrm{m}^{4}\right)$ & 105 & 3.3 \\
Porosity: $\phi$ & 0.95 & 0.98 \\
Tortuosity: $\alpha_{\infty}$ & 1 & 1.03 \\
Viscous length: $\Lambda(\mu \mathrm{m})$ & 35.1 & 78.8 \\
Thermal length $\Lambda^{\prime}(\mu \mathrm{m})$ & 105.3 & 236.4 \\
Frame density: $\rho_{1}\left(\mathrm{~kg} / \mathrm{m}^{3}\right)$ & 17 & 29 \\
\hline \hline
\end{tabular}



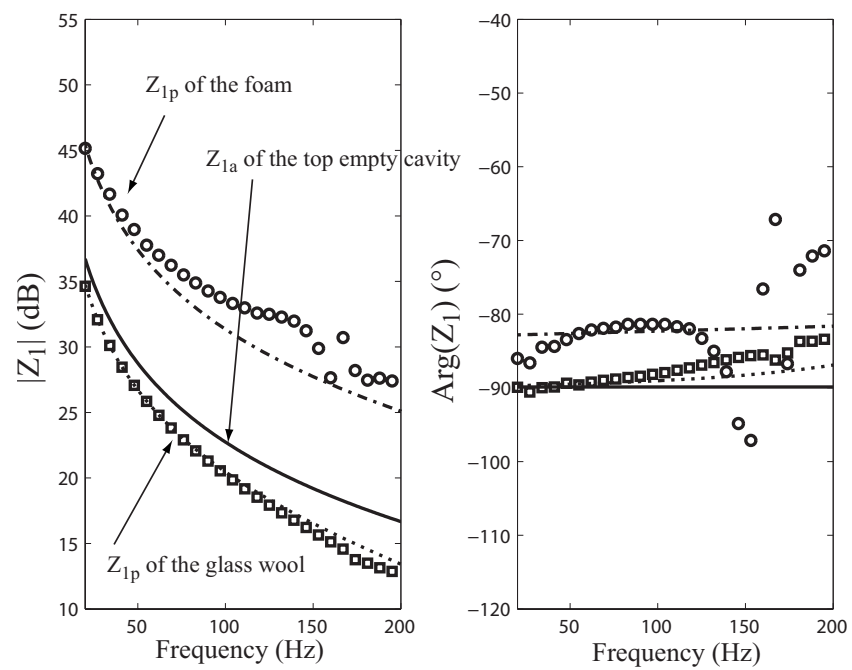

Fig. 3. Measurements (discontinuous plots) and simulations (continuous plots) of the mechanical impedance of the top empty cavity and of two porous samples: (left) magnitude, (right) phase.

moduli are considered constant in the model, which explains the discrepancy of $\left|Z_{1 p}\right|$ at low frequencies $(f<140 \mathrm{~Hz})$ in the case of the foam B: the difference increases with frequency because the Young modulus increases. ${ }^{2}$ Above $140 \mathrm{~Hz}$, measurements of the mechanical impedance $Z_{1 p}$ are not valid because of several well-known drawbacks of the loudspeaker which are not taken into account in the model: ${ }^{13}$ the stiffness of the viscoelastic suspension is nonlinear, the effect of which is paramount at the resonance frequency of the loudspeaker (around $160 \mathrm{~Hz}$ ), the inductance of the voice coil $L_{e}$ is nonconstant, and eddy currents are present in the iron-pole structure, the effects of which are predominant especially at high frequencies.

Viscoelastic properties according to frequency are finally determined by fitting the poroelastic model on the measurements. Figure 4 gives the Young's modulus and the loss factor evaluated with the classical compression quasistatic method of Fig. 1(a) and the proposed electrodynamic method for the two materials. Results determined with the electrodynamic technique are given in the linear frequency range of the loudspeaker, i.e., below $130 \mathrm{~Hz}$. Good agreements are found in the evaluation of the Young's modulus: the one of the foam is around $400 \mathrm{kPa}$ and increases with frequency; in the case of the glass wool, it is evaluated around $2.5 \mathrm{kPa}$ and is barely constant. The loss factor of the foam is also given around 0.17 by the two
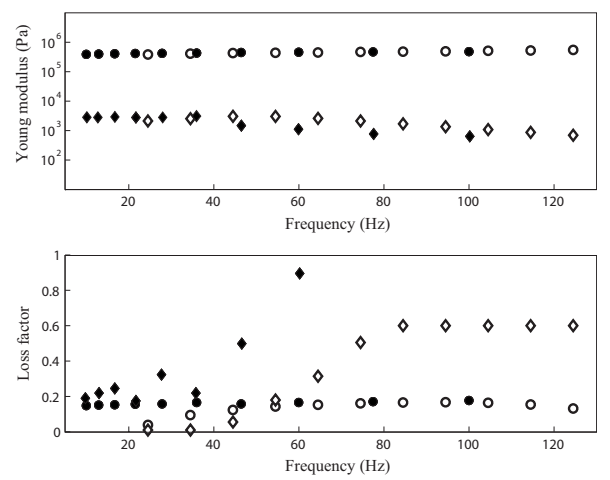

Fig. 4. Measured Young's modulus (top) and loss factor (bottom) of the glass wool (diamond) and of the polymer foam (circle): (black) classical compression quasistatic method; (white) electrodynamic method. 
methods. However, the one given by the electrodynamic method is underestimated at low frequencies. The loss factor of the glass wool estimated by the two methods does not seem realistic: ${ }^{3}$ the quasistatic method overestimates the value because of the lateral airflow, and the electrodynamic method is greatly disturbed by the electrical drawbacks of the loudspeaker, which are not accounted for in the loudspeaker modeling.

\section{Conclusion}

The feasibility to measure the viscoelastic properties of sound-absorbing materials using an electrodynamic loudspeaker has been demonstrated. The mechanical impedance of the porous sample is determined from the measurement of the electrical impedance of the loudspeaker by inverting an electroacoustic model: no additional sensors are required. Viscoelastic properties of the material are then estimated by inverting a 1D Biot model. The method has been applied using a traditional loudspeaker for frequencies below the mass-spring resonance of the system $(f<130 \mathrm{~Hz})$. Results obtained with this method are validated by comparison to the classical compression quasistatic method. The accuracy on the loss factor and the high-frequency limit could be extended with a loudspeaker free of major nonlinearities.

\section{Acknowledgments}

The authors thank the CNRS, Région Pays de la Loire and the European Commission (CREDO project) for their financial support.

\section{References and links}

${ }^{1}$ O. Doutres, N. Dauchez, and J. M. Génevaux, "Validity of the limp model for porous materials: A criterion based on the Biot theory," J. Acoust. Soc. Am. 122, 2038-2048 (2007).

${ }^{2}$ M. Etchessahar, S. Sahraoui, L. Benyahia, and J. F. Tassin, "Frequency dependence of elastic properties of acoustic foams," J. Acoust. Soc. Am. 117, 1114-1121 (2005).

${ }^{3} \mathrm{~V}$. Tarnow, "Dynamic measurements of the elastic constants of glass wool," J. Acoust. Soc. Am. 118, 3672-3678 (2005).

${ }^{4}$ T. Pritz, "Dynamic Youngs modulus and loss factor of plastic foams for impact sound isolation," J. Sound Vib. 178, 315-322 (1994).

${ }^{5}$ L. Jaouen, A. Renault, and M. Deverge, "Elastic and damping characterizations of acoustical porous materials: Available experimental methods and applications to a melamine foam," Appl. Acoust. 69, 1129-1140 (2008).

${ }^{6}$ L. Boeckx, P. Leclaire, P. Khurana, C. Glorieux, W. Lauriks, and J. F. Allard, "Investigations of the phase velocities of guided acoustic waves in soft porous layers," J. Acoust. Soc. Am. 117, 545-554 (2005).

${ }^{7}$ J.-F. Allard, B. Brouard, N. Atalla, and S. Ghinet, "Excitation of soft porous frame resonances and evaluation of rigidity coefficients," J. Acoust. Soc. Am. 121, 78-84 (2007).

${ }^{8}$ H. O. Taylor, "Tube method of measuring sound absorption," J. Acoust. Soc. Am. 24, 701-7014 (1952).

${ }^{9} \mathrm{M}$. A. Biot, "The theory of propagation of elastic waves in a fluid-saturated porous solid. I. Low frequency range. II. Higher frequency range," J. Acoust. Soc. Am. 28, 168-191 (1956).

${ }^{10}$ J. F. Allard, Propagation of Sound in Porous Media: Modelling Sound Absorbing Materials (Elsevier, New York, 1993).

${ }^{11}$ R. H. Small, “Closed-box loudspeakers systems, part 1: Analysis,” J. Audio Eng. Soc. 20, 798-808 (1972)

${ }^{12}$ C. Langlois, R. Panneton, and N. Atalla, "Polynomial relations for quasi-static mechanical characterization of isotropic poroelastic materials," J. Acoust. Soc. Am. 110, 3032-3040 (2001).

${ }^{13}$ G. Lemarquand, “Ironless Loudspeakers,” IEEE Trans. Magn. 43(8), 3371-3374 (2007). 